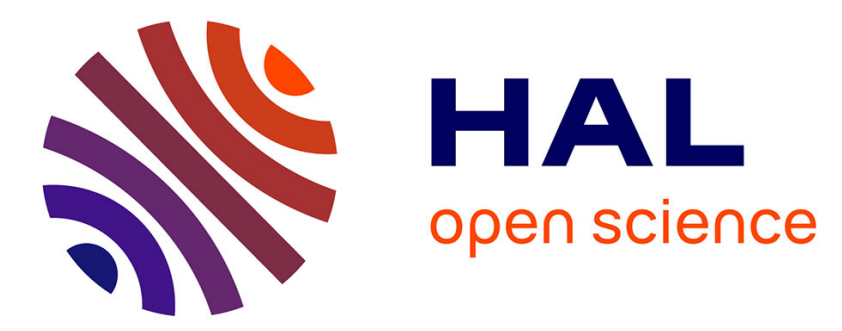

\title{
Near-field interactions between a subwavelength tip and a small-volume photonic-crystal nanocavity
}

\author{
L. Lalouat, B. Cluzel, P. Velha, E. Picard, D. Peyrade, Jean-Paul Hugonin, \\ Philippe Lalanne, E. Hadji, F. de Fornel
}

\section{- To cite this version:}

L. Lalouat, B. Cluzel, P. Velha, E. Picard, D. Peyrade, et al.. Near-field interactions between a subwavelength tip and a small-volume photonic-crystal nanocavity. Physical Review B: Condensed Matter and Materials Physics (1998-2015), 2007, 76 (4), pp.041102. 10.1103/PhysRevB.76.041102 . hal-00384580

\section{HAL Id: hal-00384580 \\ https://hal.science/hal-00384580}

Submitted on 11 Apr 2016

HAL is a multi-disciplinary open access archive for the deposit and dissemination of scientific research documents, whether they are published or not. The documents may come from teaching and research institutions in France or abroad, or from public or private research centers.
L'archive ouverte pluridisciplinaire HAL, est destinée au dépôt et à la diffusion de documents scientifiques de niveau recherche, publiés ou non, émanant des établissements d'enseignement et de recherche français ou étrangers, des laboratoires publics ou privés. 


\title{
Near-field interactions between a subwavelength tip and a small-volume photonic-crystal nanocavity
}

\author{
L. Lalouat, ${ }^{1}$ B. Cluzel, ${ }^{1,2}$ P. Velha, ${ }^{2,3,4}$ E. Picard, ${ }^{2}$ D. Peyrade,${ }^{4}$ J. P. Hugonin, ${ }^{3}$ P. Lalanne, ${ }^{3}$ E. Hadji, ${ }^{2}$ and F. de Fornel ${ }^{1}$ \\ ${ }^{1}$ Groupe d'Optique de Champ Proche-Institut Carnot de Bourgogne, UMR CNRS 5209, 9 av. Alain Savary, F-21078, DIJON, France \\ ${ }^{2}$ SiNaPS-MINATEC, CEA Grenoble, 17 rue des Martyrs, F-38054 GRENOBLE, France \\ ${ }^{3}$ Laboratoire Charles Fabry de l'Institut d'Optique, CNRS, Univ Paris-Sud, Campus Polytechnique, RD 128, \\ 91127 Palaiseau Cedex, France \\ ${ }^{4}$ LTM, CNRS, 17 rue des Martyrs, F-38054 GRENOBLE, France \\ (Received 3 June 2007; published 6 July 2007)
}

\begin{abstract}
The fundamentals of the near-field interaction between a subwavelength metallic tip and a photonic-crystal nanocavity are investigated experimentally and theoretically. It is shown experimentally that the cavity resonance is tuned without any degradation by the presence of the tip and that the reported near-field interaction is strongly related to the field distribution within the nanostructure. Then, in light of a perturbation theory, we show that this interaction is selectively related to the electric field or magnetic field distribution within the cavity, depending on the tip properties.
\end{abstract}

DOI: 10.1103/PhysRevB.76.041102

PACS number(s): 42.70.Qs, 42.50.Pq, 42.55.Sa, 42.82.-m

Recent studies have demonstrated the ability of scanning near-field optical microscopy (SNOM) techniques to evidence, below the diffraction limit, interactions between light and photonic crystals (PCs) such as coupling between spontaneous emission of quantum and the optical modes of a microcavity, ${ }^{1}$ existence of ultraslow light propagation in a photonic crystal waveguide, ${ }^{2}$ observation of Bloch harmonics ${ }^{3}$ and Bloch mode parity change in photonic crystal waveguides, ${ }^{4}$ as well as field confinement inside photonic crystal microcavities. ${ }^{5,6}$ In all these previous works, the probe was not supposed to modify the observed system. However, it has been recently observed ${ }^{7-9}$ that the nanometric tip could also be a means to change the microcavity optical properties. If, in such cases, the interaction between the near-field probe and the microcavity prevents one from a direct visualization of the electromagnetic field, it also opens the way to an exciting challenge: Using near-field probes to manipulate the light in solid-state resonators. However, to reach this objective, one needs to to bring the near-field probe in the vicinity of the resonator without introducing considerable scattering or absorption losses. Thus far, except for the theoretical predictions by Koenderink et al., such a result has never been achieved experimentally: A drastic degradation of the resonator quality factor $(Q)$ has been systematically reported as the tip was penetrating the cavity near-field. ${ }^{8,9}$

In this work, we experimentally demonstrate that a subwavelength sized metallic tip permits one to tune the resonance of an ultralow volume photonic crystal nanocavity without sacrificing its $Q$ factor. We also present an innovative mapping method, which allows us to track, at the subwavelength scale, the near-field interactions between the tip and the nanocavity. A quantitative interpretation of these interaction maps is then achieved through three-dimensional (3D) computational results associated with a perturbation theory.

Scanning electron microscope views of the nanocavity considered in this work are shown in Fig. 1(a). The cavity consists in a Fabry-Pérot-like resonator, ${ }^{10,11}$ composed of two mirrors. The nanocavity and its waveguide were realized by electron-beam lithography and inductive-coupled-plasma etching on a silicon-on-insulator substrate, having a $0.38-\mu \mathrm{m}$-thick silicon film and a $2-\mu \mathrm{m}$-thick buried oxide layer. Each mirror is formed by a periodic row of five air holes with a $180-\mathrm{nm}$ diameter and a $370-\mathrm{nm}$ periodicity, terminated by a single hole having a 140-nm-diameter at $326 \mathrm{~nm}$ from the periodic mirror. The cavity formed by the association of the two mirrors is $248 \mathrm{~nm}$ long.

In the following experiments, we use an end-fire setup to measure the cavity transmission. Light injection from a TEpolarized tunable laser source and output light collection onto an InGaAs detector are performed by using microscope objectives and monomode polarization-maintaining optical
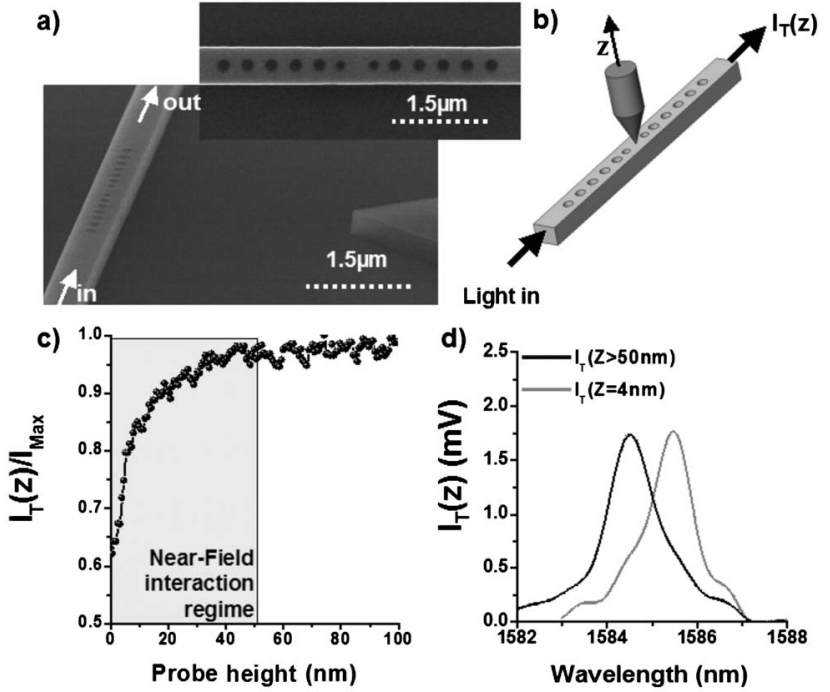

FIG. 1. (a) Scanning electron microscope views of the siliconon-insulator photonic crystal nanocavity. (b) Schematic of the experiment. (c) Cavity transmittance $I_{T}$ as a function of the tip height (z) above the center of the cavity. The input wavelength is set to the cavity resonance wavelength. (d) Cavity transmittance spectra for two different tip cavity distances, $z=4 \mathrm{~nm}$ and $z>50 \mathrm{~nm}$. 
fibers. While accurately keeping these alignments, a subwavelength sized probe ${ }^{12}$ consisting of a dielectric tip coated by a metallic $\mathrm{Cr}$-Au bilayer film is approached in the vicinity of the cavity. The tip possesses an apex diameter of $50 \mathrm{~nm}$ and is mounted on a SNOM. Thanks to a shear-force feedback, the tip is maintained at a fixed distance $(z=4 \mathrm{~nm})$ above the cavity, while piezoelectric scanners drive the lateral tip displacement.

We first identified the cavity resonance at $1584.6 \mathrm{~nm}$ (with a $Q$ factor equal to 1200) over the 400 -nm-wide photonic band gap of the mirrors. Next, the input laser wavelength is set to this cavity resonance and, as shown on Fig. 1(b), the tip scans the $z$ axis for distances ranging from 4 to $100 \mathrm{~nm}$ above the cavity while recording the cavity transmittance $I_{T}(z)$ at the same time. The transmittance is then normalized to the cavity peak transmittance in absence of the tip $\left(I_{\max }\right)$. This measurement is plotted in Fig. 1(c). For $z>50 \mathrm{~nm}$, the transmitted signal remains constant and equal to $I_{\max }$. For $z<50 \mathrm{~nm}$, the signal exponentially drops as the tip approaches the cavity surface. Such an exponential decay is characteristic of the near-field interaction between the tip and the evanescent field of the cavity mode.

Using the SNOM, the tip is then placed above the cavity at several lateral positions $(x, y)$ above the cavity and, for each position, we set successively the tip-cavity distance to $z>50 \mathrm{~nm}$ and $z=4 \mathrm{~nm}$. For each tip-cavity distance $(z)$, we recorded the corresponding cavity transmittance spectrum. In any case, as shown in Fig. 1(d), for $z>50 \mathrm{~nm}$, i.e., out of the optical near-field of the cavity, the resonance wavelength remains similar to that measured in the absence of the tip. For $z=4 \mathrm{~nm}$, we clearly observed that the presence of the tip induces a redshift of the cavity resonance wavelength in a range strongly dependent on the tip lateral position. As shown in Fig. 1(d), the maximum redshift is measured to be $0.9 \mathrm{~nm}$. Remarkably and in contrast to the previous works that use an AFM probe, ${ }^{8,9}$ we never observed any degradation of the cavity $Q$ factor or of its peak transmittance, even in the case of the strongest recorded interaction. This result confirms the theoretical prediction of Koenderink et al. We believe that our nanometric-size tip operates in a weaker interaction than the AFM probes, allowing us to achieve the cavity tuning without $Q$-factor and peak-transmittance degradation. This assumption is confirmed by the lowest redshift values obtained in this work ( $0.9 \mathrm{~nm}$ at maximum) compared to those obtained with an AFM probe $(\sim 2.3 \mathrm{~nm})$.

Then, to investigate the effect of the tip lateral position $(x, y)$ above the cavity on the reported near-field interaction, we measured the cavity transmittance at the resonance wavelength as a function of the tip position in the $(x, y)$ plane above the sample, while maintaining the tip-cavity distance at $z=4 \mathrm{~nm}$. We further refer to that procedure as an "interaction-scanning mode." Figure $2(\mathrm{~b})$ shows $I_{T}(x, y) / I_{\max }$ as a function of the $(x, y)$ tip position for two different wavelengths. The first one $(\lambda=1584.6 \mathrm{~nm})$ corresponds to the cavity resonance wavelength without interaction with the tip, $\lambda_{r}(z>50 \mathrm{~nm})$, and the second one $(\lambda=1585.5 \mathrm{~nm})$ corresponds to the cavity resonance wavelength modified by the presence of the tip. In Fig. 2(a), the topographical picture recorded at the same time as the interaction maps presented in Figs. 2(c) and 2(d) is also shown.

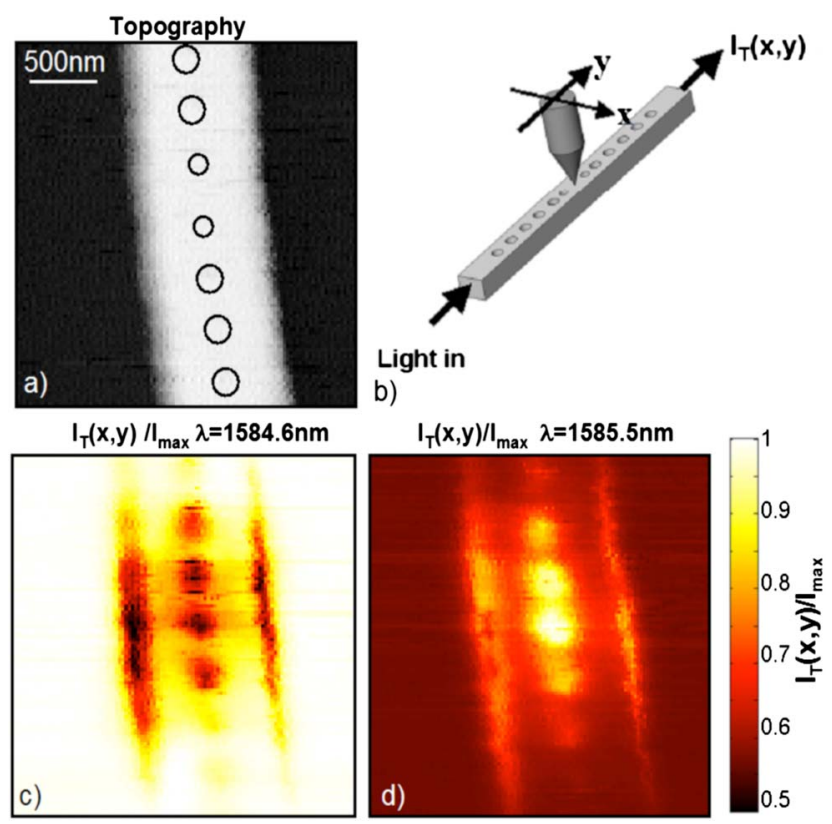

FIG. 2. (Color online) (a) Topography of the cavity recorded at the same time as the near-field interaction maps. (b) Schematic of the experiment: We record the transmittance of the cavity $I_{T}(x, y)$ as a function of the tip position $(x, y)$ with $z=4 \mathrm{~nm}$. (c) and (d) Experimental $I_{T}(x, y)$ interaction maps for two different wavelengths: (c) $\lambda_{r}(z>50 \mathrm{~nm})=1584.6 \mathrm{~nm}$ and $(\mathrm{d}) \lambda_{r}(z=4 \mathrm{~nm})=1585.5 \mathrm{~nm}$.

The interaction map clearly shows that when the tip is scanning far away from the cavity center, the transmittance is unaffected because the tip does not interact with the cavity mode. As the tip is approached near to the cavity center, the transmittance progressively decreases [see Fig. 2(c)] while it increases at $\lambda=1585.5 \mathrm{~nm}$ [see Fig. 2(d)]. Indeed, a rapid glance at the two pictures evidences that they are fully complementary. As the tip induces a redshift of the resonance, the cavity transmission $I_{T}(x, y)$ decreases at $1584.6 \mathrm{~nm}$ while it increases at $1585.5 \mathrm{~nm}$ in the same proportions: The maximum transmittance attenuation (increase) measured on Fig. 2(c) [Fig. 2(d)] is found to $-3 \mathrm{~dB}$ $(+3 \mathrm{~dB})$. This confirms that, for each position of the tip above the cavity, the peak transmittance at the resonance of the cavity-tip system stays constant even when the tip strongly interacts with the cavity mode. Moreover, the maximum redshift of the resonance associated to the $3 \mathrm{~dB}$ attenuation (or $3 \mathrm{~dB}$ increase) is consistent with the $0.9 \mathrm{~nm}$ redshift measured previously and presented in Fig. 1(d).

Then, we have analyzed the formation of the reported near-field interaction maps. Since the cavity transmittance exhibits a Lorentzian signature, the transmitted intensity through the cavity, $I_{T}\left(\lambda_{M}\right)$, at the measurement wavelength, $\lambda_{M}$, is developed as follows:

$$
I_{T}\left(\lambda_{M}\right)=\frac{A}{A^{2}+\left(\lambda_{M}-\lambda_{R_{0}}\right)^{2}},
$$

where $\lambda_{R_{0}}$ is the resonance wavelength of the cavity and $A$ is a constant that can be fitted from the transmittance spectra. 
The presence of the tip at $(x, y, z)$ above the cavity induces a resonance shift, $\delta \lambda(x, y, z)$, and the cavity resonance becomes $\lambda_{R}(x, y, z)=\lambda_{R_{0}}+\delta \lambda(x, y, z)$. Thus the transmitted intensity is

$$
I_{T}\left(x, y, z, \lambda_{M}\right)=\frac{A}{A^{2}+\left[\lambda_{M}-\lambda_{R_{0}}-\delta \lambda(x, y, z)\right]^{2}} .
$$

Next, the theory of perturbations established for microwave cavities ${ }^{13,14}$ allows us to quantify the relative wavelength shift. We consider a near-field tip with a dielectric constant $\varepsilon_{p}$ and a magnetic permeability $\mu_{p}$ above a cavity with a dielectric constant $\varepsilon$ and a magnetic permeability $\mu_{0}$ which confines an electromagnetic field $\vec{E}_{0}$ and $\vec{H}_{0}$ in a volume $V_{\text {cav }}$. As the presence of the tip above the cavity adiabatically changes the cavity resonance wavelength without introducing significant additional scattering losses (assumptions confirmed by the experimental observations), the relative wavelength shift, $\delta \lambda / \lambda$, is directly defined by the overlap integral between the unperturbed and the perturbed fields, $\vec{E}$ and $\vec{H}$, inside the perturbation volume $V_{p}$ :

$$
\frac{\delta \lambda}{\lambda}=\frac{\int_{V_{p}}\left(\vec{P}^{*} \cdot \vec{E}_{0}+\vec{M}^{*} \cdot \vec{B}_{0}\right) d v}{\int_{V_{\mathrm{cav}}}\left(\varepsilon \vec{E}_{0}^{*} \cdot \vec{E}_{0}+\mu \vec{H}_{0}^{*} \cdot \vec{H}_{0}\right) d v},
$$

where $\vec{P}$ and $\vec{M}$ are the polarization and the magnetization vectors defined as $\vec{P}=\left(\varepsilon_{p}-\varepsilon_{0}\right) \vec{E}$ and $\vec{M}=-\mu_{0} \chi_{p} \vec{H}$ with $\chi_{p}$ the magnetic susceptibility of the near-field tip. Here the denominator represents the energy stored inside the cavity volume and is independent of the perturbation as long as the unperturbed and the perturbed fields remain comparable.

This relation predicts that the wavelength shift induced by a near-field tip is entirely set by the optogeometric properties of the tip and the field distribution of the cavity mode. It also reveals that a dielectric tip perturbs the electric field of the cavity while a magnetic tip perturbs the magnetic field. Consequently, in the case of the most commonly used near-field probes made of silica, silicon, or other related materials without magnetic properties, the perturbation is entirely related to the electric field distribution above the cavity. However, if materials with a high magnetic susceptibility, such as nickel or cobalt, for instance, were used, the perturbation of the magnetic field should become predominant.

Here, our tip is coated by gold and thus its $\mu_{p}$ is close to $\mu_{0}$ and the contribution of the magnetic field in the reported interaction is negligible. Next, since the tip size is limited (which is supported by the high "resolution" of the optical pictures), its interacting volume can be modeled here as a sphere having a volume $V_{p}$. Thus, by assuming that the field inside the perturbation volume is constant, the relative wavelength shift induced by the presence of the tip is

$$
\frac{\delta \lambda}{\lambda}=\frac{3 \frac{\varepsilon_{p}-1}{\varepsilon_{p}+2}\left|\vec{E}_{0}(x, y, z)\right|^{2} V_{p}}{U},
$$

where $U$ represents the energy stored inside the cavity volume. Thus, even if our metallic possesses a negative $\varepsilon_{p}$, this
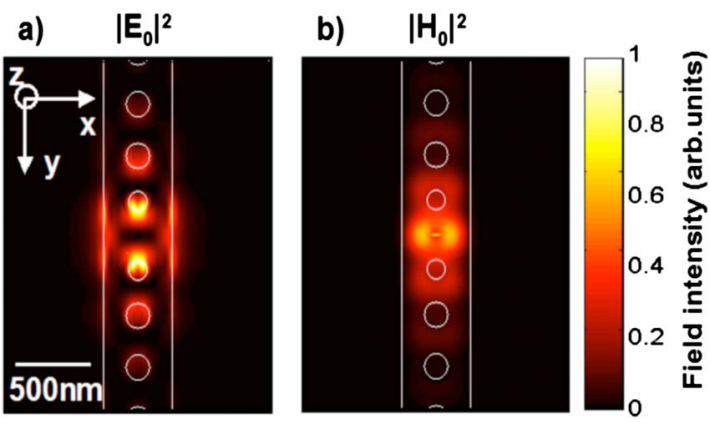

FIG. 3. (Color online) 3D calculations of the electromagnetic field distribution at $z=4 \mathrm{~nm}$ above the cavity. (a) Total electric field intensity $\left|E_{o}\right|^{2}=\left|E_{x}\right|^{2}+\left.E_{y}\right|^{2}+\left|E_{z}\right|^{2}$. (b) Total magnetic field intensity $\left|H_{o}\right|^{2}=\left|H_{x}\right|^{2}+\left.H_{y}\right|^{2}+\left|H_{z}\right|^{2}$. The different field distributions are plotted at the same scale and normalized.

implies that the cavity resonance is redshifted. Finally, using relation (2), the light transmitted through the cavity at the measurement wavelength, $\lambda_{M}$, as a function of the tip position is analytically given by

$$
I_{T}\left(x, y, z, \lambda_{M}\right)=\frac{A}{A^{2}+\left[\lambda_{M}-\lambda_{R_{0}}-D\left|\vec{E}_{0}(x, y, z)\right|^{2}\right]^{2}},
$$

where $D$ is a constant for a given tip. Thus if we consider an experimental map recorded at $\lambda_{M}=\lambda_{R_{0}}$, the intensity transmitted through the cavity is minimum where $\left|E_{0}(x, y, z)\right|^{2}$ is maximum, whereas an experimental map recorded at $\lambda_{M}$ $=\lambda_{R_{0}}+\delta \lambda_{\text {max }}$ is minimum where $\left|E_{0}(x, y, z)\right|^{2}$ is minimum.

To support this analysis, we computed the electromagnetic field distribution inside the cavity by using a 3D fully vectorial frequency-domain modal method relying on Fourier expansion techniques. ${ }^{15}$ In Fig. 3, we plotted the computed intensities of the electric and magnetic fields computed at $z=4 \mathrm{~nm}$ above the cavity: $\left|E_{O}\right|^{2}=\left|E_{x}\right|^{2}+\left.E_{y}\right|^{2}+\left|E_{z}\right|^{2}$ and $\left|H_{O}\right|^{2}=\left|H_{x}\right|^{2}+\left.H_{y}\right|^{2}+\left|H_{z}\right|^{2}$. In contrast to the study in Ref. 8, which relies on $2 \mathrm{D}$ computational results and considered an undefined "optical field," all the components of the electric field and of the magnetic fields are taken into account in this work in order to allow a quantitative comparison with the experimental near-field interaction maps. As a matter of fact, if for a TE polarization of light in a 3D asymmetric waveguide, the $x$ components of the electric field are predominant, calculations have clearly shown that the other components are clearly non-negligible: It is found here that $\left|E_{x}\right|^{2}$ is only 2.5 times larger than $\left|E_{y}\right|^{2}$ and $\left|E_{z}\right|^{2}$.

A first comparison between the reported experimental maps and the field distribution already evidences that the interaction maps are far much closer to $\left|E_{O}\right|^{2}$ than to $\left|H_{O}\right|^{2}$. This confirms the previous analysis and shows that the metallic probe used in this work perturbs selectively the electric field of the cavity.

Finally, we plotted in Fig. 4 the cross sections of the experimental map recorded at $\lambda_{M}=\lambda_{R_{0}}$ and of the cavity transmittance predicted by the relation (5) along the longitudinal and the transversal direction of the cavity (see the insets in Fig. 4). It is explicit from the figure that the theoretical and 


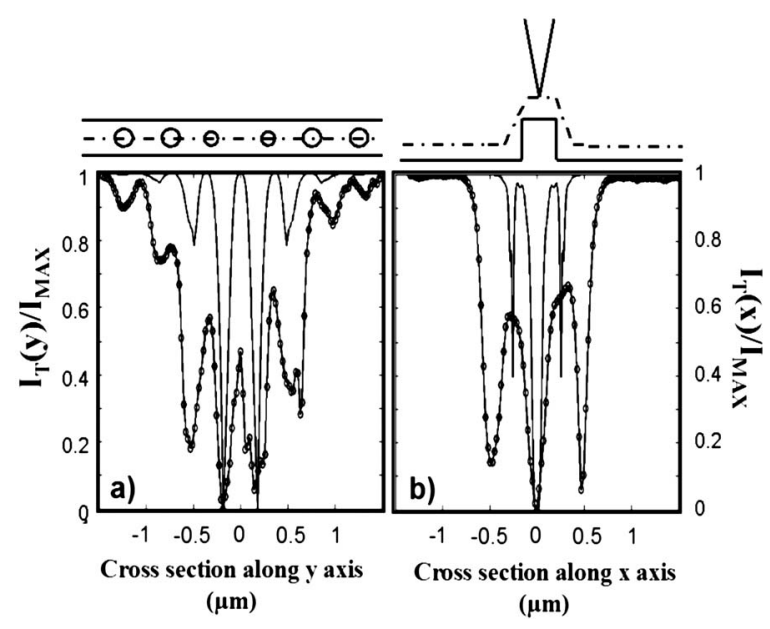

FIG. 4. Cross sections of the experimental map (dot curve) recorded at $\lambda_{M}=\lambda_{R_{0}}$ and of the cavity transmittance predicted by the relation (5) (straight curve) along the (a) longitudinal, (b) transversal directions of the cavity. The insets on the top show the positions of the cross sections and evidence the tip displacement above the cavity.

experimental results are consistent. Along the propagation axis, the 340-nm-periodicity oscillations of the transmitted intensity related to the electric field distribution above the cavity and due to the tip presence are clearly the same. The differences between the two curves are only visible in the contrast between nodes and antinodes: This corresponds to the limit of the perturbation model, which assumes that the field inside the perturbation volume is constant. As a matter of fact, even if the tip apex is limited to $50 \mathrm{~nm}$, the field inside the perturbation volume is rather averaged inside the tip than constant.

At last, along the transverse direction, the calculation and the experiment also clearly show the transverse profile of the mode propagating inside the structure. The evanescent decay of the field on each side of the waveguide, the discontinuity of the $E_{x}$ and $E_{z}$ components along the etched walls and the field confinement inside the waveguide are observed. The experimental enlargement visible on the edge of the waveguide is simply due to the scanning mode [see the inset on Fig. 4(d)]: In this region, both the tip apex and the tip side interact with the optical near-field.

To summarize, we have shown that a subwavelength tip brought in the optical near-field of a small-volume resonator permits us to finely tune its resonance without introducing additional losses. Then, we have experimentally mapped this interaction and evidenced, in light of 3D calculations associated to a perturbation theory, that the near-field interaction is strongly related to the distribution of the electric field intensity inside the cavity. Finally, the reported results also suggest that it should be possible to selectively perturb electric or magnetic fields surrounding nanocavities by controlling the near-field probe properties. This near-field effect may find several applications in the field of cavity quantum electrodynamics to finely match the cavity-atom resonances and could also open the way to an exciting challenge: The mechanical manipulation of the light at the subwavelength scale.

This work has been supported by the French ministry in charge of research with the ACI NR 63 CHABIP and by the Agence Nationale de la Recherche with the contract MIRAMAN of the French ANR Nano2006.
${ }^{1}$ N. Louvion, D. Gérard, J. Mouette, F. de Fornel, C. Seassal, X. Letartre, A. Rahmani, and S. Callard, Phys. Rev. Lett. 94, 113907 (2005).

${ }^{2}$ H. Gersen, T. J. Karle, R. J. P. Engelen, W. Bogaerts, J. P. Korterik, N. F. Van Hulst, T. F. Krauss, and L. Kuipers, Phys. Rev. Lett. 94, 073903 (2005).

${ }^{3}$ H. Gersen, T. J. Karle, R. J. P. Engelen, W. Bogaerts, J. P. Korterik, N. F. Van Hulst, T. F. Krauss, and L. Kuipers, Phys. Rev. Lett. 94, 123901 (2005).

${ }^{4}$ B. Cluzel, D. Gérard, E. Picard, T. Charvolin, V. Calvo, E. Hadji, and F. de Fornel, Appl. Phys. Lett. 85, 2682 (2004).

${ }^{5}$ P. Kramper, M. Kafesaki, C. M. Soukoulis, A. Birner, F. Müller, U. Gösele, R. B. Wehrspohn, J. Mlynek, and V. Sandoghdar, Opt. Lett. 29, 174 (2004).

${ }^{6}$ B. Cluzel, D. Gérard, E. Picard, T. Charvolin, F. de Fornel, and E. Hadji, J. Appl. Phys. 98, 086109 (2005).

${ }^{7}$ A. F. Koenderink, M. Kafesaki, B. C. Buchler, and V. Sandogh- dar, Phys. Rev. Lett. 95, 153904 (2005).

${ }^{8}$ W. C. L. Hopman, A. J. F. Hollink, R. M. de Ridder, K. O. van der Werf, V. Subramaniam, and W. Bogaerts, Opt. Express 14, 8745 (2006).

${ }^{9}$ I. Märki, M. Salt, and H. P. Herzig, Opt. Express 14, 2969 (2006).

${ }^{10}$ P. Lalanne, S. Mias, and J. P. Hugonin, Opt. Express 12, 458 (2004).

${ }^{11}$ F. Riboli, A. Recati, N. Daldosso, L. Pavesi, G. Pucker, A. Lui, S. Cabrini, and E. Di Fabrizio, Photonics Nanostruct. Fundam. Appl. 4, 41 (2006).

${ }^{12}$ Standard commercial probe product by Nanonics®.

${ }^{13}$ L. C. Maier and J. C. Slater, J. Appl. Phys. 23, 68 (1952).

${ }^{14}$ A. Gokirmak, D.-H. Wu, J. S. A. Bridgewater, and S. M. Anlage, Rev. Sci. Instrum. 69, 3410 (1998).

${ }^{15}$ E. Silberstein, P. Lalanne, J. P. Hugonin, and Q. Cao, J. Opt. Soc. Am. A 18, 2865 (2001). 